\title{
Cryptosporidiosis - A Plausible Cause for Relapse of Guillain-Barré Syndrome
}

\author{
Asadullah Anees Khan ${ }^{1}$, Karthik Somasundaram ${ }^{1}$ \\ 1. Intensive Care Unit, St. Peter's Hospital, Ashford and St. Peter's Hospitals NHS Trust, Chertsey, GBR
}

Corresponding author: Asadullah Anees Khan, auakhan@hotmail.com

\begin{abstract}
A 25-year-old female presented on the acute medical take with rapidly evolving ascending weakness, sensory loss, and areflexia after a prodromal diarrhoeal illness, ultimately critical care admission, tracheostomy, and intravenous immunoglobulin (IVIG) therapy. The patient had been diagnosed with Guillain-Barré Syndrome (GBS) six years previously, treated with intravenous Immunoglobulin, and discharged after a five-day in-patient stay without mechanical ventilation. On this occasion, a diagnosis of recurrent GBS was made, supported by cytoalbuminological dissociation in the cerebrospinal fluid (CSF). Investigations for infective precipitants were negative aside from a stool culture, positive for Cryptosporidium spp. DNA (deoxyribonucleic acid) two weeks earlier. There are no previously reported cases of GBS due to cryptosporidiosis on PubMed.
\end{abstract}

The patient was treated with a course of IVIG and discharged from critical care after 66 days, requiring ongoing neurorehabilitation, which is likely to be prolonged.

Categories: Internal Medicine, Neurology, Infectious Disease

Keywords: guillain barre syndrome (gbs), guillain-barre syndrome (gbs), acute flaccid paralysis, bilateral limb weakness, cryptosporidiosis, gastroenteritis, id critical care, traveller medicine, relapse, neurology and critical care

\section{Introduction}

Guillain-Barré Syndrome (GBS) is a well-recognised demyelinating polyneuropathy that is preceded by a prodromal illness in two-thirds of cases. Recurrent episodes are uncommon but occur in a subset of patients, being commoner in patients below 30 years of age [1]. Causative organisms include Campylobacter jejuni, Hepatitis viruses, Cytomegalovirus (CMV), Mycoplasma, Epstein-Barr virus (EBV), Haemophilus influenzae, and others $[2,3]$. Our literature review identified that cryptosporidiosis has not previously been identified as a precipitant of GBS.

Review began 04/17/2021 Review ended 04/19/2021 Published 04/23/2021

\section{๑) Copyright 2021}

Khan et al. This is an open access article distributed under the terms of the Creative Commons Attribution License CC-BY 4.0., which permits unrestricted use, distribution, and reproduction in any medium, provided the original author and source are credited.

\section{Case Presentation}

A 25-year-old female presented with distal sensory loss in the upper limbs and gradual onset progressive ascending weakness over 3-4 days.

She suffered from a diarrhoeal illness recently, having returned from Pakistan four weeks before presentation to hospital. Stool cultures sent from primary care two weeks before admission returned positive for Cryptosporidium spp. DNA (deoxyribonucleic acid) polymerase chain reaction (PCR) and she was treated with ciprofloxacin for this. Testing for common infective precipitants including Campylobacter was carried out on the same sample however returned a negative result. Her presenting symptoms followed a paediatric admission for her 13-month-old daughter's following a similar bout of gastroenteritis. The daughter did not undergo stool culture testing and no precipitating organism was identified in her case.

The patient had experienced an episode of acute Guillain-Barré Syndrome six years previously with rapid recovery at another institution. On that occasion, she required one course of intravenous immunoglobulin (IVIG) leading to improvement and never experienced respiratory failure. The only other past medical history of note was gestational diabetes mellitus.

On examination, she had symmetric ascending flaccid paresis with weakness and areflexia. Flexion and extension movements at the hip, knee, and ankle were MRC (Medical Research Council) Grade 2/5 bilaterally with MRC Grade 3/5 at the wrist and 4/5 at the elbow as well as the shoulder. The total MRC-sum score on admission was 36/60. On presentation, due to a lack of facial weakness, she had a total EGRIS (Erasmus GBS Respiratory Insufficiency Score) score of 4 which predicted a 32\% chance of respiratory insufficiency during the first week of admission. She was physiologically stable when admitted, however, was moved to critical care for monitoring of FVC (forced vital capacity). Twelve hours into her admission, she developed a weak voice and with a rising oxygen requirement and was subsequently intubated and mechanically ventilated for progressive respiratory failure. 
Admission blood tests were within normal limits, however, the inflammatory markers (white cell count and C-reactive protein) proceeded to rise in the first two days of admission. Treatment was instituted for aspiration pneumonia due to the visualisation of pooled saliva in the airway pre-intubation. Nasogastric feeding (NG) was commenced as a prolonged period of ventilation was anticipated and the patient had already demonstrated cranio-bulbar signs.

A CT Brain on day 1 of her admission did not reveal any abnormalities and she underwent full neuraxial imaging on day 3 of admission including magnetic resonance scanning of the brain and spinal cord with contrast - these did not reveal an abnormal pathology either and were within normal limits. She underwent a lumbar puncture on day 1 of admission which revealed evidence of cyto-albuminological dissociation (Table 1).

\begin{tabular}{|c|c|c|}
\hline Test & Result & Range \\
\hline Appearance & Clear, colourless & \\
\hline Red blood cells (last sample) & $<1 \times 10^{6} / \mathrm{L}$ & \\
\hline White blood cells & $<1 \times 10^{6} / \mathrm{L}$ & \\
\hline Organisms & No organisms seen & \\
\hline Culture & No growth after 48 hours & \\
\hline Protein & $0.53 \mathrm{~g} / \mathrm{L}$ & $0.15-0.4$ \\
\hline Oligoclonal bands Albumin (CSF) & $0.37 \mathrm{~g} / \mathrm{L}$ & $0.00-0.17$ \\
\hline Oligoclonal bands IgG (CSF) & $0.04 \mathrm{~g} / \mathrm{L}$ & $0.000-0.017$ \\
\hline
\end{tabular}

\section{TABLE 1: Lumbar Puncture Results}

CSF - cerebrospinal fluid

She was reviewed by the neurologist and investigations for HIV, hepatitis viruses, Epstein-Barr virus (EBV), Cytomegalovirus (CMV), ANA (Hep-2), GM1 Abs, GQ1B Abs, Anti-MPO, and Anti-PR as well as Lyme disease and syphilis were negative. Nerve conduction studies were performed and demonstrated in-excitability (described as absent distal motor responses or only present in one nerve with very low amplitude) of peripheral motor nerves and normal sensory nerves, in keeping with the Guillain-Barré Syndrome spectrum.

Treatment was commenced with IVIG at a dose of $0.4 \mathrm{~kg} /$ bodyweight for five days. This was followed by an IVIG course two weeks after the first course. A tracheostomy was sited on day 4 of admission. She remained in critical care for 66 days until she had demonstrated sufficient improvement in peripheral muscular function and recovery of strength in her pharyngeal muscles. Her admission was complicated by low mood and neuropathic pain which were treated with citalopram and pregabalin respectively. She was discharged to the ward after decannulation, and the NG tube was removed after being cleared by the SALT (Speech and Language Team) with ongoing physiotherapy support. Her recovery is expected to be prolonged.

Three weeks after moving to the respiratory ward, she was discharged to a neuro-rehabilitation unit where she stayed for two months. She has now been discharged to her own home, where she is still improving and has regained use of her upper limbs, walking with crutches. She still requires regular physiotherapy.

\section{Discussion}

This is the first reported trigger of GBS secondary to cryptosporidiosis. Relapse of GBS is not common reportedly only 1 - 3\% of cases [4]. Relapses are also milder than the original illness, common in patients below 30 years of age, and usually Miller-Fisher variants. [5] Recurrent GBS is also not necessarily associated with a prodrome and can be resistant to immunomodulatory treatment as in this case where the patient required two courses of IVIG and without rapid or complete resolution of symptoms to date.

There are four main mechanisms thought to lead to an immune response responsible for the signs and symptoms of Guillain-Barré Syndrome [6]: 1. Antibody mediated - Serum antibodies to peripheral nerves (Anti-GM1, Anti-GD1a, Anti-G1b, etc.) are demonstrable in 50\% of patients [6]; 2. Molecular mimicry - The most likely mechanism due to evidence of Campylobacter infection leading to GBS, certain lipo-

oligosaccharides react with gangliosides in nervous tissue, these anti-bodies are also cross-reactive [6]; 3. Complement mediated - Due to complement activation at the nerve site. There is some evidence for the 
complement inhibitor eculizumab inhibited neurotoxicity [6]; 4. Patient susceptibility - Certain factors in individuals might make them more susceptible to infection, therefore increasing the risk of developing GBS.

Cryptosporidium is a parasitic alveolate that is known to cause mostly gastrointestinal, biliary, and respiratory infections in humans [7]. This parasite has a peculiar ability to colonise many epithelial surfaces and has been identified in the pancreas as well as the urinary bladder as mentioned by Sponseller et al. [7]. This is more common in immunodeficient hosts (e.g., with HIV) [8], but it can also cause a self-limiting illness in the immunocompetent [8]. There are still 4000 cases annually in England and Wales despite a reduction in reported cases [9]. The illness can spread by contacting infected animals or drinking contaminated water. In developing countries, 8-19\% of diarrhoeal illnesses can be attributed to cryptosporidiosis [10].

There is a plausible case for Cryptosporidium parvum to be responsible for immunologic phenomena in humans. As we know, Campylobacter jejuni exhibits molecular mimicry [11] - the lipopolysaccharides on $C$. jejuni react with gangliosides in nerve cells causing the formation of anti-ganglioside antibodies (which are detectable in sera of patients). This molecular mimicry leads to neuronal destruction and subsequent neurological weakness. Some reports have suggested cases of GBS associated with Campylobacter have a worse prognosis due to the severity of the autoimmune response causing axonal injury [11,12]. In a similar vein, antibodies in humans and animals with cryptosporidiosis react with antigenic proteins (CApy and $\mathrm{CP} 15$ ) on the organism's surface, making an immunological response likely. Currently, this has only been studied experimentally in mice models [13]; further research and awareness are needed in human hosts. These same antigenic targets are being investigated in order to develop a vaccine.

This episode of neurological weakness is GBS and not CIDP (chronic inflammatory demyelinating polyneuropathy) due to the following reasons: There was an acute course preceded by prodromal illness (progression over days as opposed to weeks and months which is common in CIDP); the presence of respiratory failure - this is uncommon in CIDP as opposed to classic GBS (AIDP, etc.); improvement with IVIG and no requirement for steroid therapy; as well as no previous relapses since the initial episode five years ago.

Extra-intestinal manifestation of Cryptosporidial infection has been previously described. Several studies have shown that infection with Cryptosporidium hominis is linked to extra-intestinal sequelae like arthralgias, ocular symptoms, dizziness, headache, and malaise [14].

\section{Conclusions}

To conclude we would like to highlight the following points: Cryptosporidium has not been known to cause Guillain-Barré Syndrome but should be recognized as a potential trigger. Patients presenting with weakness and reduced/absent tendon reflexes with a prodromal infective aetiology should be tested for cryptosporidiosis if usual precipitants are not identified - diarrhoeal illnesses that cause GBS may be undiagnosed cryptosporidiosis. A history of previous GBS does not rule out further relapses of GBS. Although uncommon, GBS can recur at any point with variable sequalae.

\section{Additional Information \\ Disclosures}

Human subjects: Consent was obtained or waived by all participants in this study. Conflicts of interest: In compliance with the ICMJE uniform disclosure form, all authors declare the following: Payment/services info: All authors have declared that no financial support was received from any organization for the submitted work. Financial relationships: All authors have declared that they have no financial relationships at present or within the previous three years with any organizations that might have an interest in the submitted work. Other relationships: All authors have declared that there are no other relationships or activities that could appear to have influenced the submitted work.

\section{References}

1. Kuitwaard K, van Koningsveld R, Ruts L, Jacobs BC, van Doorn PA: Recurrent Guillain-Barré syndrome. J Neurol Neurosurg Psychiatry. 2009, 80:56-9. 10.1136/jnnp.2008.156463

2. van den Berg B, Walgaard C, Drenthen J, Fokke C, Jacobs BC, van Doorn PA: Guillain-Barré syndrome: pathogenesis, diagnosis, treatment and prognosis. Nat Rev Neurol. 2014, 10:469-82. 10.1038/nrneurol.2014.121

3. Hughes RAC, Cornblath DR, Willison HJ: Guillain-Barré syndrome in the 100 years since its description by Guillain, Barré and Strohl. Brain. 2016, 139:3041-7. 10.1093/brain/aww247

4. Almadani A, Alboudi A, Alrukn S, Sarathchandran P, Inshasi J: Recurrent Guillain Barre Syndrome- a report of three cases with clinical electrophysiological and CSF profile (P2.070). Neurology. 2015, 84:2.070.

5. Hadden RD: Deterioration after Guillain-Barré syndrome: recurrence, treatment-related fluctuation or CIDP?. J Neurol Neurosurg Psychiatry. 2009, 80:3. 10.1136/jnnp.2008.160069

6. van Doorn PA, Kuitwaard K, Walgaard C, van Koningsveld R, Ruts L, Jacobs BC: IVIG treatment and prognosis in Guillain-Barré syndrome. J Clin Immunol. 2010, 30 Suppl 1:S74-8. 10.1007/s10875-010-9407-4

7. Sponseller JK, Griffiths JK, Tzipori S: The evolution of respiratory cryptosporidiosis: evidence for 


\section{Cureus}

transmission by inhalation. Clin Microbiol Rev. 2014, 27:575-86. 10.1128/CMR.00115-13

8. Wang ZD, Liu Q, Liu HH, Li S, Zhang L, Zhao YK, Zhu XQ: Prevalence of Cryptosporidium, microsporidia and Isospora infection in HIV-infected people: a global systematic review and meta-analysis. Parasit Vectors. 2018, 11:28. 10.1186/s13071-017-2558-X

9. McKerr C, Chalmers RM, Vivancos R, O'Brien SJ, Mugarza J, Christley RM: Cross-sectional investigation of household transmission of Cryptosporidium in England and Wales: the epiCrypt study protocol. BMJ Open. 2019, 9:e026116. 10.1136/bmjopen-2018-026116

10. Bouzid M, Kintz E, Hunter PR: Risk factors for Cryptosporidium infection in low and middle income countries: a systematic review and meta-analysis. PLoS Negl Trop Dis. 2018, 12:e006553.

10.1371/journal.pntd.0006553

11. Islam A, Abraham S, Moran AP: Campylobacter jejuni-mediated Guillain-Barre syndrome, an overview of the molecular mimicry and vaccine development approaches. J Neurol Neuroscience. 11,

12. Poropatich KO, Walker CL, Black RE: Quantifying the association between Campylobacter infection and Guillain-Barré syndrome: a systematic review. J Health Popul Nutr. 2010, 28:545-52. 10.3329/jhpn.v28i6.6602

13. Bartelt LA, Bolick DT, Kolling GL, et al.: Cryptosporidium priming is more effective than vaccine for protection against cryptosporidiosis in a murine protein malnutrition model. PLoS Negl Trop Dis. 2016, 10:e0004820. 10.1371/journal.pntd.0004820

14. Cama VA, Bern C, Roberts J, et al.: Cryptosporidium species and subtypes and clinical manifestations in children, Peru. Emerg Infect Dis. 2008, 14:1567-74. 10.3201/eid1410.071273 